\title{
SOME GEOMETRIC ASPECTS OF HYPERBOLIC BOUNDARY VALUE PROBLEMS
}

\author{
J. BRIAN CONREY AND MICHAEL W. SMILEY
}

(Communicated by Barbara L. Keyfitz)

\begin{abstract}
Let $L$ denote the linear operator associated with the wave equation when it is subjected to boundary conditions in both space and time. The properties of invertibility or partial invertibility of $L$, and compactness of the (partial) inverse when it exists, are characterized in terms of the space time domain $\Omega \times(0, T)$, for all rectangular domains $\Omega \subset \mathbf{R}^{n}$.
\end{abstract}

In the study of boundary value problems for the wave equation, features of the underlying spatial domain play a significant role. The purpose of this note is to describe some of these features, and to characterize their influence on the problem. In particular we shall describe the influence of the set $\Omega$ on the boundary value problem

$$
\begin{aligned}
u_{t t}-\Delta u+\sigma u & =f, & (t, x) \in \mathbf{R} \times \Omega, \\
u(t, x) & =0, & (t, x) \in \mathbf{R} \times \partial \Omega, \\
u(t+T, x) & =u(t, x), \quad & (t, x) \in \mathbf{R} \times \Omega,
\end{aligned}
$$

in which $\sigma \in \mathbf{R}$, and $f: \mathbf{R} \times \Omega \rightarrow \mathbf{R}$ is $T$-periodic in $t$ and square-integrable over $(0, T) \times \Omega$. We will be interested in determining properties of the associated linear operator $L$. Of primary interest is the property of invertibility or partial invertibility, and if $L$ has this property compactness or noncompactness of the (partial) inverse will also be of interest.

In the above problem, the partial differential equation is of hyperbolic type, and the surfaces $t=$ constant are noncharacteristic. Normally the data $u(0, x)$ $=u_{0}(x), u_{t}(0, x)=u_{1}(x)$ would be prescribed on the surface $t=0$ in place of $(0.3)$, and in this case the problem would be a well-posed initial-value problem. By well-posed we mean (cf. [9]) that the problem has a unique solution which depends continuously on the data, consisting of $u_{0}, u_{1}$, and $f$. In sharp contrast, the time-periodic problem posed above, in which boundary conditions

Received by the editors July 10, 1988 and, in revised form, December 12, 1988.

1980 Mathematics Subject Classification (1985 Revision). Primary 11E25, 35L05, 35L20.

Key words and phrases. Wave equation, linear operator, spectrum, cluster point, quadratic form, Fredholm operator, compact operator.

The research of Conrey was supported in part by the Institute for Advanced Study, Princeton, New Jersey. 
are prescribed on the noncharacteristic surfaces, is almost always ill-posed. In some cases there are infinitely many solutions, and in other cases there may not be any solutions (which are square-integrable). There are a few cases in which the problem is well-posed.

The time-periodic problem $(0.1)-(0.3)$, despite these unpleasant mathematical aspects, is of considerable interest as a physical model. In a sense it represents an infinite dimensional Hamiltonian system. The above problem also represents a simplified version of the corresponding semilinear problem, in which $(0.1)$ is replaced by

$$
u_{t t}-\Delta u+g(\dot{u})=f, \quad(t, x) \in \mathbf{R} \times \Omega .
$$

This problem has been considered by several authors (cf. [3]) in the recent past, mostly when $\Omega=(0, a)$ is an interval. In this case the associated linear operator $L \sim\left[\partial_{t}^{2}-\partial_{x}^{2},(0.2)-(0.3)\right]$ has an infinite dimensional null space, when the ratio $T / a$ is rational; and has a trivial null space but is not onto when $T / a$ is an algebraic number of degree 3 or greater (cf. [18]). Usually the problem is dealt with under the assumption that $T / a$ is rational (often $T=2 \pi, a=\pi$ ), and in this case a Fredholm alternative applies.

Historically, problem $(0.1)-(0.3)$ was first considered from a classical perspective (cf. [2,9]), and the Dirichlet conditions, $u(0, x)=u(T, x)=0$, were used in place of the periodic conditions (0.3). As is pointed out below, there is no significant difference between the Dirichlet and the periodic problem, when considered in the framework of $\S 1$. Subsequent authors (cf. [4, 6, 8, $11,18]$ ) used generalized solutions defined in various ways. It is the work of the second author [18] that provides the basis for the work here. Thus by a solution we mean a distributional solution, relative to the set of test functions $\left\{\varphi \in C^{\infty}(\mathbf{R} \times \Omega): \varphi(t+T, x)=\varphi(t, x)\right.$ for all $(t, x) \in \mathbf{R} \times \Omega$, and $\left.\varphi(t, \cdot) \in C_{0}^{\infty}(\Omega)\right\}$. Here $C_{0}^{\infty}$ denotes the set of smooth functions having compact support in $\Omega$. In the cited work, a spectral condition is used to determine the nature of the operator $L$ alluded to above. Here we discuss the influence of the spatial domain $\Omega$ on the spectrum of $L$. Even when the geometry is simple there are a variety of possibilities, and so even in these cases a complete resolution of some of the questions will require some recent results from the theory of numbers.

In the first section we briefly describe the framework set out in [18] and pose the questions we will subsequently address. In the next section we give the needed number theoretic results, and in the final section we use these results to provide answers to the questions raised at the outset. In addition we give some results regarding the nonlinear problem $\left(0.1^{\prime}\right),(0.2)-(0.3)$.

\section{ABSTRACT HYPERBolic BOUNDARY VALUE PROBLEMS}

Following [18] we let $H$ denote a real separable Hilbert space, with norm and inner product $(\cdot, \cdot)$. We use $L$ to denote a closed symmetric (not necessarily bounded) operator in $H$ which satisfies the hypotheses: 
$\left(L H_{1}\right) \quad L$ has eigenvalues $\left\{\lambda_{i}: i \in I\right\}$ and eigenvectors (i.e. functions) $\left\{\varphi_{i}: i \in I\right\}$, where $I$ denotes a countable index set. Thus $\left(L \varphi_{i}, u\right)=\lambda_{i}\left(\varphi_{i}, u\right), \forall i \in I, \forall u \in H$.

$\left(L H_{2}\right)$ The set $\left\{\varphi_{i}: i \in I\right\}$ is a complete orthonormal system in $H$. Let $I_{0}=\left\{i \in I: \lambda_{i}=0\right\}$ and $I_{1}=\left\{i \in I: \lambda_{i} \neq 0\right\}$. If $I_{0} \neq \varnothing$, the empty set, then we can only hope to invert $L$ on $H_{1}=\operatorname{ker}(L)^{\perp}$. This can be done when 0 is not a cluster point of the nonzero spectrum $\Lambda_{1}=\left\{\lambda_{i}: i \in I_{1}\right\}$; and in this case there is a duality theory, analogous to the Lax-Milgram theory for elliptic boundary value problems, that applies. If 0 is a cluster point of $\Lambda_{1}$ then in fact $L$ is not onto $H_{1}$. In this case it is still possible to obtain distributional solutions $u \sim \sum \lambda_{i}^{-1} f_{i} \varphi_{i}$, however for certain $f \in H_{1}$ these solutions will not have $\|u\|<+\infty$; hence they do not belong to $H$.

Theorem 1.1. If there exists $\delta>0$ such that $(-\delta, \delta) \cap \Lambda_{1}=\varnothing$, then:

(i) There exists a Hilbert space $\mathscr{H}_{1} \subset H_{1}$, with dual $\mathscr{H}_{1}^{*}$, such that the inclusions $\mathscr{H}_{1} \subset H_{1} \subset \mathscr{H}_{1}^{*}$ are continuous, dense, and injective.

(ii) The operator $L$ is an isometric isomorphism from $\mathscr{H}_{1}$ onto $\mathscr{H}_{1}^{*}$.

(iii) The operator $L$ has a continuous partial inverse $K: \mathscr{H}_{1}^{*} \rightarrow \mathscr{H}_{1}$ satisfying $L K=i d_{\mathscr{P}_{1}}$ and $K L=i d_{\mathscr{P}_{1}}$. Moreover, using $K$ to also denote the restriction of $K$ to $H_{1}$, we have that $K: H_{1} \rightarrow \mathscr{H}_{1}$ is continuous with $L K=i d_{H_{1}}$ and $L K=i d_{\mathscr{H}_{1}}$ on $\operatorname{dom}(L)=\left\{u \in \mathscr{H}_{1}: L u=f\right.$ for some $\left.f \in H_{1}\right\}$.

Remark. We say $L u=f \in H_{1}$ if and only if $\left(u, L \varphi_{i}\right)=\left(f, \varphi_{i}\right)$ for all $i \in I_{1}$.

There are three situations that may arise when the above theorem is applicable. It may happen that $\operatorname{ker}(L)=\{0\}$ so that in fact $L$ is invertible with a continuous inverse. It may happen that $\operatorname{ker}(L)$ is finite-dimensional, in which case $L$ is a Fredholm operator (with index zero) in the sense of [10] and has the partial inverse $K$ described above. A third alternative is that $\operatorname{ker}(L)$ is infinite-dimensional, and again has the partial inverse $K$ described above. In this case we will call $L$ quasi-Fredholm.

When considering $(0.1)-(0.3)$ in the context of the above framework, and assuming $\Omega=(0, a)$ with $T / a$ rational, one finds that such a $\delta>0$ exists and that $K$ is in fact compact as a map from $H_{1}$ into $\mathscr{H}_{1}$. Here one has $H=L^{2}((0, T) \times(0, a))$. It is also known that $K: H_{1} \rightarrow L^{\infty}((0, T) \times(0, a))$ is compact (cf. $[3,12])$. Not surprisingly this property is exploited in dealing with $\left(0.1^{\prime}\right),(0.2)-(0.3)$. In general compactness can be characterized by a finiteness condition on the spectrum (cf. [10]).

Theorem 1.2. Suppose that there is a $\delta>0$ such that $(-\delta, \delta) \cap \Lambda_{1}=\varnothing$, and let $K$ denote the (partial) inverse of $L$. Then $K$ is compact if and only if each $\lambda_{i} \in \Lambda_{1}$ has finite multiplicity and each of the sets $\Lambda_{1} \cap[-n, n]$ is finite.

Remark. From the proof of this result (cf. [18]), it is easy to see that $K$ is compact from $H_{1}$ into $\mathscr{H}_{1}$ if and only if it is compact from $H_{1}$ into $H_{1}$. 
The above results allow us to discuss certain aspects of the linear operator $L$ associated with problem $(0.1)-(0.3)$. Let $\ell=\left(\ell_{1}, \cdots, \ell_{n}\right)$ be a multi-index, with each $\ell_{i} \in\{1,2,3, \ldots\}$, and define $|\ell|=\ell_{1}+\cdots+\ell_{n}$. If $\left\{\mu_{\ell}\right\}_{|\ell| \geq n}$ denotes the set of eigenvalues for the problem

$$
\begin{aligned}
\Delta v+\mu v & =0, & & x \in \Omega, \\
v(x) & =0, & & x \in \partial \Omega,
\end{aligned}
$$

then the eigenvalues corresponding to the operator $L$ are $\lambda_{k \ell}=\mu_{\ell}-(2 \pi k / T)^{2}+$ $\sigma$. Clearly, if the time-periodic boundary conditions $(0.3)$ are replaced by Dirichlet, Neumann or mixed boundary conditions, these values and their multiplicities do not significantly change their nature with regard to the applicability of the above theorems. By investigating the appropriateness of the hypotheses, in these theorems, we shall address the following questions:

(i) What is the dimension of $\operatorname{ker}(L)$ ?

(ii) Does Theorem 1.1 apply, so that $L$ is at least partially invertible? If this is the case, the answer to the first question determines whether $L$ is invertible or partially invertible, and if it's partially invertible whether it's Fredholm of quasi-Fredholm as defined above.

(iii) When $L$ is invertible, or partially invertible, is the map $K$ compact?

Obviously, the answers to these questions have important implications for developing a theory applicable to the corresponding nonlinear problems. Each of these questions translates directly into a question regarding the distribution of the eigenvalues $\lambda_{k \ell}$, and the number of solutions of equations of the form $\lambda_{k \ell}=\lambda$. As a special case we focus our attention on the example of a parallelepiped $\Omega=\prod_{i=1}^{n}\left(0, \alpha_{i} \pi\right) \subset \mathbf{R}^{n}$. With $T=2 \pi$ we have

$$
\lambda_{k \ell}=\left(\frac{\ell_{1}}{\alpha_{1}}\right)^{2}+\cdots+\left(\frac{\ell_{n}}{\alpha_{n}}\right)^{2}-k^{2}+\sigma
$$

for $k \geq 0$ and $\ell_{i} \geq 1,1 \leq i \leq n$. Of course $\alpha_{i}>0$ for $1 \leq i \leq n$.

\section{ON THE RANGE OF THE ASSOCIATED QUADRATIC FORM}

In this section we let $F\left(x_{1}, \ldots, x_{n}, y\right)=\beta_{1} x_{1}^{2}+\cdots+\beta_{n} x_{n}^{2}-y^{2}$, where $\beta_{i}>0$ for $1 \leq i \leq n$. We are interested in the set of values $F$ takes on as the $x_{i}$ range through the positive integers and $y$ ranges through the nonnegative integers. Thus we define $\Gamma=F\left((\mathbf{Z}-\{0\})^{n}, \mathbf{Z}\right)$ to be the range of $F$. Our assertions about $\Gamma$ depend in general on $n$ and on the nature of the $\beta_{i}$.

We let $\mathbf{Q}$ denote the set of rational numbers; $\mathbf{Q}^{2}$ is the set of squares of rational numbers and $\mathbf{Q}^{1 / 2}=\{\alpha+\beta \sqrt{\delta}: \alpha, \beta, \delta \in \mathbf{Q}\}$ is the set of numbers which satisfy a quadratic equation with rational coefficients. We let $\mathbf{B}$ denote the set of 'badly approximable' numbers $\alpha$ such that if $\alpha=\left[a_{0} ; a_{1}, a_{2}, \ldots\right]$ is the continued fraction expansion of $\alpha$ then $\sup \left\{a_{m}: m \geq 0\right\}<\infty$. B is an uncountable set of measure 0 . We note that $\mathbf{Q}^{2} \subset \mathbf{Q} \subset \mathbf{Q}^{1 / 2} \subset \mathbf{B} \subset \mathbf{R}$, with all 
inclusions proper, and that the only easily obtained examples of numbers in $\mathbf{B}$ that are not in $\mathbf{Q}$ are those in $\mathbf{Q}^{1 / 2}-\mathbf{Q}$.

An alternative definition of $\mathbf{B}$ which explains the term 'badly approximable' is $\mathbf{B}$ is the set of $\alpha \in \mathbf{R}$ for which there exists a $c(\alpha)>0$ such that the inequality $|\alpha-a / q|<c(\alpha) / q^{2}$ has no solutions in integers. From this definition, it is easy to see that $\alpha \in \mathbf{B}-\{0\}$ if and only if $1 / \alpha \in \mathbf{B}$. For, suppose $\alpha \in \mathbf{B}-\{0\}$, and $a, q \in \mathbf{Z}, q \neq 0$. If $|a / q|>2 /|\alpha|$, then

$$
|1 / \alpha-a / q|>1 /|\alpha| \geq 1 / \alpha q^{2} \mid \text {. }
$$

If $|a / q| \leq 2 /|\alpha|$, then

$$
|1 / \alpha-a / q|=|\alpha-q / a||a| /|q \alpha| \geq c(\alpha) /|\alpha q a| \geq c(\alpha) /\left(2 q^{2}\right) .
$$

Thus, with $c(1 / \alpha)=\min \{1 /|\alpha|, c(\alpha) / 2\}$ we see that $1 / \alpha \in \mathbf{B}$.

In the lemmas that follow $x_{i}$ and $y$ will always denote integers, and $\beta_{i}$ will always denote a positive number. In the event that $n=1$ we will use $\beta$ and $x$ in place of $\beta_{1}$ and $x_{1}$. When we speak of an equation having a nontrivial solution, we mean a solution where the variables are integers and not all of them are zero. A positive solution will mean a solution in which all variables are positive integers. The symbol $\gamma$ will be reserved for a nonzero element of $\Gamma$ and by the multiplicity of $\gamma$ we mean the number of solutions of $F=\gamma$ with $x_{i} \geq 1, y \geq 0$. Note that by the homogeneity of $F$, if $0 \in \Gamma$ then it has infinite multiplicity.

Lemma 2.1. Suppose that $n=1$. If $\beta \in \mathbf{B}$ then $\Gamma$ is a discrete subset of $\mathbf{R}$. If $\beta \notin \mathbf{B}$, then $\Gamma$ is dense in $\mathbf{R}$.

This result is well known and may be found in Niven and Zuckerman [17].

Lemma 2.2. If $n \geq 2$ and $\beta_{i} \notin \mathbf{Q}$ for some $i$, then $\Gamma$ is dense in $\mathbf{R}$.

Proof. Recently Margulis $[13,14]$ has shown that if $Q$ is any real, indefinite quadratic form in $m \geq 3$ variables, which is not a scalar multiple of a form with all rational coefficients, then $Q\left(\mathbf{Z}^{m}\right)$ is a dense subset of $\mathbf{R}$. We have essentially the same situation here except that our $x_{i}$ are not allowed to be 0 . Without loss of generality we assume that $\beta_{1} \notin \mathbf{Q}$. The case $n>2$ of our lemma follows trivially from the case $n=2$, so we assume now that $n=2$. Also if either $\beta_{1} \notin$ $\mathbf{B}$ or $\beta_{2} \notin \mathbf{B}$, then the lemma follows from Lemma 2.1. Hence we assume that $\beta_{1}, \beta_{2} \in \mathbf{B}$ with $\beta_{1} \notin \mathbf{Q}$. Now $F\left(\mathbf{Z}^{2}, \mathbf{Z}\right)=\Gamma \cup F(0, \mathbf{Z}, \mathbf{Z}) \cup F(\mathbf{Z}, 0, \mathbf{Z})$. By Margulis' theorem, $F\left(\mathbf{Z}^{2}, \mathbf{Z}\right)$ is dense in $\mathbf{R}$. By Lemma 2.1 both $F(0, \mathbf{Z}, \mathbf{Z})$ and $F(\mathbf{Z}, 0, \mathbf{Z})$ are discrete. Therefore $\Gamma$ must be dense.

Lemma 2.3. Suppose that $n=1$.

(i) If $\beta \in \mathbf{Q}^{2}$, then $0 \in \Gamma$ and each $\gamma$ has finite multiplicity.

(ii) If $\beta \in \mathbf{Q}-\mathbf{Q}^{2}$, then $0 \notin \Gamma$ and if $F(x, y)=\delta$ has a nontrivial solution, then it has infinitely many positive solutions. Hence each $\gamma$ has infinite multiplicity.

(iii) If $\beta \notin \mathbf{Q}$, then $0 \notin \Gamma$ and each $\gamma$ has multiplicity one. 
Proof. (i) Let $\beta=c^{2} / d^{2}$ where $c$ and $d$ are positive coprime integers. Then $(x, y)=(d m, c m), m \in \mathbf{Z}^{+}$, gives infinitely many positive solutions to $F(x, y)$ $=0$. If $F(x, y)=\gamma \neq 0$, then

$$
|F(x, y)|=\left|\frac{c}{d} x-y\right|\left(\frac{c}{d} x+y\right) \geq d^{-1}\left(\frac{c}{d} x+y\right) \rightarrow+\infty,
$$

as $x+y \rightarrow \infty$. Thus there can be at most finitely many positive solutions in this case.

(ii) Let $\beta=c / d$ with $c$ and $d$ positive coprime integers. Since $\beta \notin \mathbf{Q}^{2}$, it is clear that $F(x, y)=0$ has no solution. The other assertion follows from a general theorem of Gauss (cf. Mordell [16, Chapter 8, Theorem 2]). (To apply this, note that $d \delta \in \mathbf{Z}$ and $c d$ is not a square since $\beta \notin \mathbf{Q}^{2}$.)

(iii) If $F\left(x_{1}, y_{1}\right)=F(x, y)$, then $\beta\left(x^{2}-x_{1}^{2}\right)=y^{2}-y_{1}^{2}$. Then $\beta \notin \mathbf{Q}$ implies that $x^{2}=x_{1}^{2}$ and $y^{2}=y_{1}^{2}$. Since the variables are nonnegative it follows that $x=x_{1}$ and $y=y_{1}$.

We now consider the case of $n>1$. Several lemmas will be needed to obtain the analogue of Lemma 2.2. We begin with the question of multiplicity.

Lemma 2.4. If $n>2$ and $\beta_{i} \in \mathbf{Q}$ for $1 \leq i \leq n$, then each $\gamma$ has infinite multiplicity.

Proof. We consider two cases. First suppose that $\beta_{i} \notin \mathbf{Q}^{2}$ for some $i$. Without loss of generality we may assume that $\beta_{1} \notin \mathbf{Q}^{2}$. Suppose that $F\left(x_{1}^{*}, \ldots, x_{n}^{*}\right.$, $\left.y^{*}\right)=\gamma$ with $x_{i}^{*} \geq 1$ for $1 \leq i \leq n$. Let $\gamma^{*}=\beta_{2} x_{2}^{* 2}+\cdots+\beta_{n} x_{n}^{* 2}$. Then $\left(x_{1}^{*}, y^{*}\right)$ is a nontrivial solution of $\beta_{1} x_{1}^{2}-y^{2}=\gamma-\gamma^{*}$, so that by Lemma 2.3(ii) this equation has infinitely many positive solutions $\left(x_{1}, y\right)$. But for each such $\left(x_{1}, y\right)$ we get a positive solution $\left(x_{1}, x_{2}^{*}, \ldots, x_{n}^{*}, y\right)$ of $F\left(x_{1}, \ldots, x_{n}, y\right)=\gamma$. Hence, in this case $\gamma$ has infinite multiplicity.

In the other case $\beta_{i} \in \mathbf{Q}^{2}$ for $1 \leq i \leq n$. We initially consider $n=2$ here. After clearing denominators we see that it suffices to prove that

$$
a^{2} x_{1}^{2}+b^{2} x_{2}^{2}-c^{2} y^{2}=N
$$

has infinitely many positive solutions assuming that it has at least one solution; here $a, b, c \in \mathbf{Z}^{+}$and $N \in \mathbf{Z}$. Let $\left(x_{1}^{*}, x_{2}^{*}, y^{*}\right)$ be a solution of $(2.1)$ and let $r=-2 b c\left(b x_{2}^{*}-c y^{*}\right)$. We will prove that for infinitely many $t \in \mathbf{Z}^{+}$there is a solution $\left(x_{1}, x_{2}, y\right)$ of $(2.1)$ with

$$
x_{2}=x_{2}^{*}+a^{2} c t, \quad y=y^{*}+a^{2} b t \quad(r \geq 0),
$$

or

$$
x^{2}=-x_{2}^{*}+a^{2} c t, \quad y=-y^{*}+a^{2} b t \quad(r<0) .
$$

Indeed, substituting (2.2) or (2.3) into (2.1) and simplifying we obtain

$$
x_{1}^{2}-x_{1}^{* 2}=|r| t \text {. }
$$


If $r=0$, then (2.4) is satisfied by $x_{1}=x_{1}^{*}$ and any $t \in \mathbf{Z}^{+}$. If $r \neq 0$, then (2.4) still has infinitely many positive solutions as can be seen by letting $t=2|r| u^{2}$ and observing that the resulting equation

$$
x_{1}^{2}-2 r^{2} u^{2}=x_{1}^{* 2}
$$

has infinitely many positive solutions by virtue of Lemma 2.3(ii), since $2 r^{2} \notin$ $\mathbf{Q}^{2}$ and $x_{1}=x_{1}^{*}, u=0$ is a nontrivial solution. Now note that if $r \geq 0$ then for any positive solution $\left(x_{1}, u\right)$ of (2.5) we obtain from (2.2) a positive solution $\left(x_{1}, x_{2}, y\right)$ of $(2.1)$; if $r<0$, then any positive solution $\left(x_{1}, u\right)$ of (2.5) with $u$ sufficiently large yields a positive solution $\left(x_{1}, x_{2}, y\right)$ of $(2.1)$ via (2.3). But it is clear that only finitely many of the solutions of (2.5) have $u$ smaller than any given bound. This completes the proof when $n=2$.

Now suppose that $n>2$. Let $F\left(x_{1}^{*}, \ldots, x_{n}^{*}, y^{*}\right)=\gamma$ with $x_{i}^{*} \geq 1$ for $1 \leq i \leq n$. Let $\gamma^{*}=\beta_{3} x_{3}^{* 2}+\cdots+\beta_{n} x_{n}^{* 2}$.

Then

$$
\beta_{1} x_{1}^{2}+\beta_{2} x_{2}^{2}-y^{2}=\gamma-\gamma^{*}
$$

has a nontrivial solution $\left(x_{1}^{*}, x_{2}^{*}, y^{*}\right)$. If $\gamma-\gamma^{*}=0$ we see that $F\left(t x_{1}^{*}, t x_{2}^{*}\right.$, $\left.x_{3}^{*}, \ldots, x_{n}^{*}, t y^{*}\right)=\gamma$ for any $t \in \mathbf{Z}^{+}$so that $\gamma$ has infinite multiplicity. If $\gamma-\gamma^{*} \neq 0$, then the above argument for the case $n=2$ leads to infinitely many positive solutions $\left(x_{1}, x_{2}, y\right)$ of (2.6). For each of these, we have

$$
F\left(x_{1}, x_{2}, x_{3}^{*}, \ldots, x_{n}^{*}, y\right)=\gamma
$$

so that $\gamma$ has infinite multiplicity. This completes the proof of the lemma.

Note that if $n=1$ and $\beta \in \mathbf{Q}^{2}$ the assertion of Lemma 2.4 is false, as follows from Lemma 2.3(i). We next consider the question of whether $0 \in \Gamma$. We begin with an auxiliary result.

Lemma 2.5. Suppose that $\beta_{i} \in \mathbf{Q}$ for $1 \leq i \leq n$. If $F\left(x_{1}, \ldots, x_{n}, y\right)=0$ has a nontrivial solution, then it has a positive solution and $0 \in \Gamma$.

Proof. We proceed by induction on $n$. The assertion when $n=1$ follows from Lemma 2.3. Assume the assertion true for $n-1$ and suppose that $\left(x_{1}^{*}, \ldots, x_{n}^{*}, y^{*}\right)$ is a nontrivial solution in the case $n$. By the induction hypothesis we may assume that $x_{1}^{*}=0$ and $x_{i}^{*}>0$ for $2 \leq i \leq n$. Clearly $y^{*}>0$. It suffices to find a positive solution $(x, y)$ of

$$
\beta_{1} x_{1}^{2}-y^{2}=-y^{* 2}
$$

for then $\left(x, x_{2}^{*}, \ldots, x_{n}^{*}, y\right)$ will be a positive solution of $F\left(x_{1}, \ldots, x_{n}, y\right)=0$.

We now consider two cases. If $\beta_{1} \notin \mathbf{Q}^{2}$, then (2.7) has (infinitely many) positive solutions by Lemma $2.3(\mathrm{ii})$, since it has the nontrivial solution $\left(0, y^{*}\right)$. In the second case suppose that $\beta_{1}=c^{2} / d^{2}$ with $c$ and $d$ positive coprime integers. Now by the homogeneity of $F\left(x_{1}, \ldots, x_{n}, y\right)$ we may assume that 
in our solution $\left(x_{1}^{*}, \ldots, x_{n}^{*}, y^{*}\right)$ we have $y^{*}=2 c y$ for some $y>1$. Then $(x, y)=\left(d\left(y^{2}-1\right), c\left(y^{2}+1\right)\right)$ is a positive solution of $(2.1)$.

The question of whether or not $0 \in \Gamma$ is subtle in the cases $n=2$ and 3 and some $\beta_{i} \notin \mathbf{Q}^{2}$. In order to explain this situation more easily we formulate the following.

Definition. Suppose that all $\beta_{i} \in \mathbf{Q}$. Then the standard form of $F$ is the quadratic form obtained from $F$ by applying the following algorithm.

(i) Multiply $F$ by $d=1$.c. m. $\left[d_{1}, \ldots, d_{n}\right]$, where $d_{i}$ is the denominator of $\beta_{i}$, to obtain $F^{(1)}$.

(ii) If $F^{(1)}=a_{1} x_{1}^{2}+\cdots+a_{n} x_{n}^{2}-b y^{2}$ with $a_{i}, b \in \mathbf{Z}^{+}$, then replace $b$ and each $a_{i}$ by its squarefree part. (If $m \in \mathbf{Z}^{+}$, then the squarefree part of $m$ is $m / d^{2}$ where $d$ is the maximum integer such that $d^{2} \mid m$.)

(iii) Let $F^{(2)}=a_{1} x_{1}^{2}+\cdots+a_{n} x_{n}^{2}-b y^{2}$ denote the form obtained after (ii). Let $g=$ g. c.d. $\left(a_{1}, a_{2}, \ldots, a_{n},-b\right)$. Divide each coefficient of $F^{(2)}$ by $g$.

(iv) Let $F^{(3)}=a_{1} x_{1}^{2}+\cdots+a_{n} x_{n}^{2}-b y^{2}$ denote the form obtained after (iii). If there is a prime $p$ which divides $n$ of the $n+1$ coefficients then divide each of these coefficients by $p$ and multiply the remaining coefficient by $p$. Repeat this step until no $n$ of the $n+1$ coefficients have a common prime factor.

Lemma 2.6. Suppose that all $\beta_{i} \in \mathbf{Q}$. If $n \geq 4$ or if all $\beta_{i} \in \mathbf{Q}^{2}$, then $0 \in \Gamma$. If $n=2$ or 3 and some $\beta_{i} \notin \mathbf{Q}^{2}$ then $0 \in \Gamma$ if and only if all of the following conditions are satisfied:

$n=2:$ Let $G=a x_{1}^{2}+b x_{2}^{2}-c y^{2}$ be the standard form of $F$.

$C(2): G\left(x_{1}, x_{2}, y\right) \equiv 0 \bmod 8$ has a solution with at least one variable odd.

$C(p)$ : For all permutations $(\alpha, \beta, \gamma)$ of $(a, b, c)$ if $p$ is an odd prime such that $p \mid \alpha$ then $-\beta \gamma$ is a square modulo $p$.

$n=3:$ Let $G=a x_{1}^{2}+b x_{2}^{2}+c x_{3}^{2}-d y^{2}$ be the standard form of $F$.

$C(2): G\left(x_{1}, x_{2}, x_{3}, y\right) \equiv 0 \bmod 8$ has a solution with at least one variable odd.

$C(p)$ : For all permutations $(\alpha, \beta, \gamma, \delta)$ of $(a, b, c, d)$ if $p$ is an odd prime such that $p \mid \alpha$ and $p \mid \beta$, then either $-\gamma \delta$ is a square modulo $p$ or $-\alpha \beta / p^{2}$ is a square modulo $p$.

Proof. The case where all $\beta_{i} \in \mathbf{Q}^{2}$ may be found in Smiley [19]. For the other cases, we see by Lemma 2.5 that $0 \in \Gamma$ if and only if $F\left(x_{1}, \ldots, x_{n}, y\right)=0$ has a nontrivial solution. But if $n \geq 4$, then $F=0$ has a nontrivial solution and if $n=2$ (resp. $n=3$ ) then $F=0$ has a nontrivial solution if and only if the local conditions $C(2)$ and $C(p)$ (for all odd primes $p$ ) are satisfied. This last 
statement is a consequence of the Hasse-Minkowski local to global principal for quadratic forms and may be found in Mordell [16, Chapter 7] or Borevich and Shafarevich [1, Chapter 1].

We summarize the results of this section in the following.

Theorem 2.1. If $n=1$ and $\beta \in \mathbf{B}$, or if $n \geq 2$ and all $\beta_{i} \in \mathbf{Q}$, then $\Gamma$ is $a$ discrete subset of $\Gamma$. In all other cases $\Gamma$ is dense in $\mathbf{R}$. Furthermore, when $\mathbf{R}$ is discrete we have the following situations:

(1) If $n=1$ then the following cases arise:

(i) If $\beta \in \mathbf{Q}^{2}$ then $0 \in \Gamma$ and it has infinite multiplicity; each $\gamma$ has finite multiplicity.

(ii) If $\beta \in \mathbf{Q}-\mathbf{Q}^{2}$ then $0 \notin \Gamma$ and each $\gamma$ has infinite multiplicity.

(iii) If $\beta \in \mathbf{B}-\mathbf{Q}$ then $0 \notin \Gamma$ and each $\gamma$ has multiplicity one.

(2) If $n \geq 2$ and all $\beta_{i} \in \mathbf{Q}$, then each $\gamma$ has infinite multiplicity. If $n \geq 4$, then $0 \in \Gamma$. If $n=2$ or 3 , then $0 \in \Gamma$ if and only if the conditions of Lemma 2.6 are satisfied. In particular if all $\beta_{i} \in \mathbf{Q}^{2}$ then $0 \in \Gamma$. If $0 \in \Gamma$, then it has infinite multiplicity.

\section{INVERTIBILITY AND COMPACTNESS OF THE ASSOCIATED OPERATORS}

Throughout this section we assume $T=2 \pi$ and $\Omega=\prod_{i=1}^{n}\left(0, \alpha_{i} \pi\right) \subset \mathbf{R}^{n}$. As in $\S 1$ we use $L$ to denote the closed symmetric linear operator associated to $(0.1)-(0.3)$, and $\lambda_{k \ell}$ to denote the eigenvalues of $L$ as given in (1.3). Let $\sigma+\Gamma=\left\{\lambda_{k \ell}: k \geq 0, \ell_{i} \geq 1\right.$ for $\left.1 \leq i \leq n\right\}$; it should be clear that $\Gamma$ has the same meaning as in $\S 2$, with the understanding that $\beta_{i}=\alpha_{i}^{-2}$. When it exists we use $K$ to denote the (partial) inverse of $L$. Portions of the following theorems, in which $n=1$ and $\alpha \notin \mathbf{Q}$ or when $n \geq 2$, have previously been obtained by McKenna [15] and Smiley [18,19].

Theorem 3.1. If $n=1$ and $\alpha^{2} \notin \mathbf{B}$, or if $n \geq 2$ and some $\alpha_{i}^{2} \notin \mathbf{Q}$, then $\sigma+\Gamma$ is dense in $\mathbf{R}$. In all other cases $\sigma+\Gamma$ is discrete, and hence Theorem 1.1 applies.

This follows immediately from Lemmas 2.1 and 2.2. According to Theorems 1.1 and 1.2 the results of the previous section have the following consequence.

Theorem 3.2. Suppose $\sigma=0$.

(1) If $n=1$ and $\alpha^{2} \in \mathbf{B}$ then the following cases arise:

(i) If $\alpha \in \mathbf{Q}$ then $L$ is quasi-Fredholm and $K$ is compact.

(ii) If $\alpha^{2} \in \mathbf{Q}-\mathbf{Q}^{2}$ then $L$ is invertible and $K$ is not compact.

(iii) If $\alpha^{2} \in \mathbf{B}-\mathbf{Q}$ then $L$ is invertible and $K$ is compact.

(2) If $n \geq 2$ and $\alpha_{i}^{2} \in \mathbf{Q}$ for $1 \leq i \leq n$ then $L$ is either invertible or quasiFredholm, and $K$ is always noncompact. $L$ is quasi-Fredholm if $n \geq 4$ or when 
$n=2$ or 3 and the conditions of Lemma 2.6 are satisfied. In particular $L$ is quasi-Fredholm when $\alpha_{i} \in \mathbf{Q}$ for each $i$.

Whenever Theorem 1.1 applies, one can also show that solutions of the semilinear problem $\left(0.1^{\prime}\right),(0.2)-(0.3)$ exist, under appropriate hypotheses on $g: \mathbf{R} \rightarrow \mathbf{R}$. This is true regardless of the dimension of $\operatorname{ker}(L)$. In any case we assume $g \in C(\mathbf{R})$, and without loss of generality we can also assume that $g(0)=0$. Furthermore $g$ should satisfy:

$\left(\mathrm{G}_{1}\right)$ There is a constant $\beta_{1}>0$ such that $|g(u)-g(v)| \leq \beta_{1}|u-v|$, for all $u, v \in \mathbf{R}$.

We may also require:

$\left(\mathrm{G}_{2}\right)$ There is a constant $\beta_{0}>0$, with $\beta_{0} \leq \beta_{1}$, such that $[g(u)-g(v)](u-$ $v) \geq \beta_{0}|u-v|^{2}$, for all $u, v \in \mathbf{R}$.

The inequality of $\left(G_{1}\right)$ guarantees that $g$ generates a Lipschitz continuous Nemytsky operator (cf. [20]) from $H=L^{2}((0, T) \times \Omega)$ into itself, while $\left(\mathrm{G}_{2}\right)$ would imply that the operator is strongly monotone. Under the hypothesis of Theorem 3.2, we can define numbers $\mathscr{K}=\inf \left\{\left|\lambda_{k \ell}\right|: \lambda_{k \ell}<0\right\}$ and $\delta=$ $\inf \left\{\left|\lambda_{k \ell}\right|: \lambda_{k \ell} \neq 0\right\}$. Clearly $0<\delta \leq \mathscr{K}$.

Theorem 3.3. Suppose that $n=1$ and $\alpha^{2} \in \mathbf{B}$, or that $n \geq 2$ and $\alpha_{i}^{2} \in \mathbf{Q}$ for $1 \leq i \leq n$. If $\operatorname{ker}(L)=\{0\}$ and $g$ satisfies $\left(G_{1}\right)$ with $\beta_{1}<\delta$, or if $\operatorname{dim}(\operatorname{ker}(L))>0$ and $g$ satisfies $\left(G_{1}\right)$ and $\left(G_{2}\right)$ with $\beta_{1}^{2}<\mathscr{K} \beta_{0}$ then problem $\left(0.1^{\prime}\right)$, (0.2)-(0.3) has a unique solution $u \in L^{2}((0, T) \times \Omega)$ for every $f \in$ $L^{2}((0, T) \times \Omega)$. Moreover $u$ depends continuously on $f$.

This result follows directly from the abstract theory presented in [19]. The proof in the case $\operatorname{ker}(L)=\{0\}$ follows standard arguments. In the other case one employs the method of alternative problems, and splits the problem into two parts. The property of monotonicity is used in a crucial way to handle the part associated to the kernel of $L$. We mention that it is the monotonicity and not the sign of the monotonicity that is crucial. The result still follows when $g$ is monotone decreasing; one then defines $\mathscr{K}=\inf \left\{\lambda_{k \ell}: \lambda_{k \ell}>0\right\}$.

The inequalities of Theorem 3.2 imply that $g(u)$ is a nonlinear perturbation whose linearization at the origin $\gamma u$ is such that $\gamma>0$ is small relative to the proximity of $\Lambda_{1}$ to the origin. If $\gamma$ is large then we should replace $g(u)$ by $h(u)=g(u)-\sigma u$, with $\sigma$ near $\gamma$ (and $\sigma<\gamma$ ), and consider

$$
u_{t t}-\Delta u+\sigma u+h(u)=f, \quad(t, x) \in \mathbf{R} \times \Omega,
$$

together with $(0.2)-(0.3)$. A theorem similar to the one above can then be stated. Toward a description of the linearized problem in this case we have the following.

Corollary 3.4. Suppose that $n=1$ and $\alpha^{2} \in \mathbf{B}$ or $n \geq 2$ and $\alpha_{i}^{2} \in \mathbf{Q}$ for $1 \leq i \leq n$. Assume that $\sigma \neq 0$. 
If $-\sigma \notin \Gamma$ then $L$ is invertible. If $-\sigma \in \Gamma$ then $L$ is quasi-Fredholm except when $n=1$ and either $\alpha \in \mathbf{Q}$ or $\alpha^{2} \in \mathbf{B}-\mathbf{Q}$; in these two cases $L$ is Fredholm. The operator $\mathscr{K}$ is not compact except for the one case in which $n=1$ and $\alpha^{2} \in \mathbf{B}-\mathbf{Q}$.

\section{REFERENCES}

1. Z. I. Borevich and I. R. Shafarevich, Number theory, Pure and Applied Mathematics, Vol. 20, Academic Press, New York, 1966.

2. D. G. Bourgin and R. J. Duffin, The Dirichlet problem for the vibrating string equation, Bull. Amer. Math. Soc. 45(1939), 851-858.

3. H. Brezis, Periodic solutions of nonlinear vibrating strings and duality principles, Bull. Amer. Math. Soc. 8(3)(1983), 409-426.

4. F. E. Browder, A remark on the Dirichlet problem for non-elliptic self-adjoint partial differential operators, Rend. Circ. Mat. Palermo, 6(2)(1957), 249-253.

5. _ - On the Dirichlet problem for linear non-elliptic partial differential equations II, Rend. Circ. Mat. Palermo, 7(2)(1958), 303-308.

6. D. R. Dunninger, The Dirichlet problem for a nonhomogeneous wave equation, Boll. Un. Math. Ital., 2-A(6)(1983), 253-265.

7. D. R. Dunninger and H. A. Levine, Uniqueness criteria for solutions to abstract boundary value problems, J. Differential Equations 22(6)(1976), 368-378.

8. D. W. Fox and C. Pucci, The Dirichlet problem for the wave equation, Ann. Mat. Pura Appl. 46(4)(1958), 155-182.

9. J. Hadamard, Equations aux derivees partielles, Enseign. Math. 35(1936), 5-42.

10. T. Kato, Perturbation theory for linear operators, 2nd. ed., Springer-Verlag, New York, 1976.

11. I. S. Louhivaara, Uber das Dirichletsche problem fur die selbstadjungierten linearen partiellen Differentialgleichungen zweiter Ordnung, Rend. Circ. Mat. Palermo 5(2)(1956), 260-274.

12. H. Lovicarova, Periodic solutions of a weakly nonlinear wave equation in one dimension, Czech. Math. J. 19(1969), 324-342.

13. G. A. Margulis, Formes quadratrigues indefinies et flots unipotents sur les espaces homogènes, C. R. Acad. Sci. Paris Ser. I. Math. 304(1987), no. 10, 249-253.

14. _ Indefinite quadratic forms and unipotent flows on homogeneous spaces, (to appear in Semester on Dynamical Systems and Ergodic Theory, Warsaw 1986 Banach Center Publications).

15. P. J. McKenna, On solutions of a nonlinear wave question when the ratio of the period to the length of the interval is irrational, Proc. Amer. Math. Soc. 93(1)(1985), 59-64.

16. L. J. Mordell, Diophantine equations, Pure and Applied Mathematics, Vol. 30, Academic Press, New York, 1977.

17. I. Niven and H. S. Zuckerman, The theory of numbers, 4th ed., Wiley, New York, 1980.

18. M. W. Smiley, Hyperbolic Boundary Value Problems. A Lax-Milgram approach and the vibrating string, Boll. Un. Mat. Ital. 5-A(6)(1986), 23-32.

19. ___ Eigenfunction mests and nonlinear hyperbolic boundary value problems at resonance, J. Math. Anal. Appl. 122(1)(1987), 129-151.

20. M. Vainberg, Variational methods for the study of nonlinear operators, (translated by A. Feinstein), Holden-Day, San Francisco, 1964.

Department of Mathematics, Oklahoma State University, Stillwater, Oklahoma 74074. The Institute for Advanced Study, Princeton, New Jersey 08540.

Department of Mathematics, Iowa State University, Ames, Iowa 50011 